\title{
A Novel Approach for Solving Engineering Optimization Problems Involving Equality Constraints
}

\author{
Kedar Nath Das and Tapan Kumar Singh
}

\begin{abstract}
In general, the optimization problems arise in real life situations are constrained in nature. Handling such problems becomes more difficult when an optimization problem involves some equality constraints. The computation complexity increases exponentially if the constraints are non-linear and non-convex. In order to solve such problems, a large number of evolutionary approaches have been suggested in the literature. In such approaches, penalty function method and boundary simulation method are more popular to solve equality and inequality constraints problems. In this paper, an efficient and novel approach namely 'Drosophila Food-Search Constrained Optimization Algorithm (DFCOA)' has been proposed that helps for the robust generation of feasible regions during simulation. The proposed algorithm is initially tested on ten typical constrained benchmark problems with different tastes. Further two real life engineering problems have also been solved. The simulation results confirm that the proposed algorithm works better than some of the state-of-the-art algorithms.
\end{abstract}

Index Terms-Tournament selection, mQA, redundant search, GPCR.

\section{INTRODUCTION}

Development of robust algorithms to solve complex optimization problems has been one of the most important issues in the branch of Computer science. Mostly, traditional methods become handicapped in solving such complex structured problems. In such cases, Evolutionary Algorithm (EA) is an alternate paradigm.

The global optimization algorithms have been widely applied in many fields of science and engineering. The main concern of these methods is to find the global optimal solution over the existence of several local optima in the systems. In searching global optimal solution, EAs work on a set of individuals (called population) by repeated application of their stochastic operators. However, the presence of equality constraints in the optimization problem makes it more complex. Eventually, the involvement of bit constraint violation in the optimal solution obtained by EAs is unavoidable.

Some of the most popular EAs suggested in the literature are Genetic Algorithm (GA) [1], Particle Swarm Optimization (PSO) [2], Simulated Annealing algorithm (SA) [3], Cultural Evolutionary algorithm [4], Modified Differential Evolution (COMDE) [5], Rough Penalty Genetic Algorithm (RPGA) [6], Modified Artificial Bee Colony algorithm (MABC) [7] etc. However, most of these

Manuscript received February 9, 2015; revised April 24, 2015.

The authors are with the Department of Mathematics, NIT Silchar, Assam, India (e-mail: kedar.iitr@gmail.com,tksingh1977.nits@gmail.com). algorithms are either complicated or suffer with computational burdensome to handle equality constraints. Moreover, most of them are involved with some of the parameters to fine tune. Therefore, in this paper, a new algorithm called Drosophila Food-Search Constrained Optimization Algorithm (DFCOA) algorithm is presented. DFCOA is based on the food search behavior of a fruit fly namely 'Drosophila Melanogaster'. DFCOA comprises of Drosophila Food-Search Optimization (DFO) algorithm [8] with modified penalty method for constraint handling.

The rest of paper organized as follows. Section II presents a discussion about Drosophila Melanogaster. Section III introduces constrained handling technique. In Section IV, the proposed Drosophila Food-Search Constrained Optimization Algorithm is introduced. Section V comprises of the computational results for benchmark problems. Section VI presents the results of real life problems. Section VII concludes the present study with highlighting the future scope of research.

\section{DRosophila Melanogaster}

Drosophila Melanogaster (Fig. 1) is a fly consisting of many sensory systems including taste and olfaction [9]-[10]. Drosophila consists with proboscis (Fig. 2), wings, legs and ovipositor [10].The body of a fly surrounded by a hair like structure called Sensilla. The sensilla are of two types (a) internal and (b) external sensilla. The external sensilla have to search preferable food sources and internal sensilla analyze the foods before allowed into digestive system. Further, internal sensilla used either as sensor for harmful substances or to verify substances for sucking. The Drosophila ingest food through it proboscis. The taste bristles and pegs have a terminal pore at the tip to allow direct access of food substances to the dendrite process of the gustatory receptor neuron (GRNs).

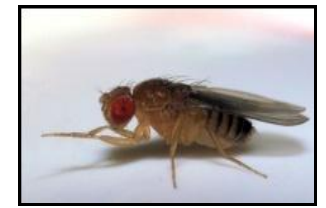

Fig. 1. Drosophila Melanogaster.

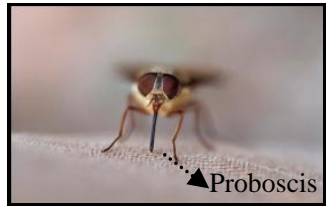

Fig. 2. Gustatory receptors.
The Drosophila also consisting with olfactory receptors [11] in which 60 Drosophila olfactory receptors (DOR) genes encode a family of G-Protein-Coupled-receptors, whose function is to recognize odorant molecules. Each olfactory receptor neuron (ORN) expressed as a single odorant receptor. The optimized Food-Search behavior of Drosophila 
has been modeled in to optimization algorithm and has been successfully used to solve unconstrained optimization problem in [8]. The beauty of this algorithm is it is robust and is parameter free. In this paper, the code is developed in to handle constraints. Special care has been taken to deal with equality constraints too.

\section{CONSTRAined HANDLING TECHNIQUE}

In last few decades many techniques have been developed by the researches for handling of constraints. According to Michalewicz [12], Eiben [13] and Coello [14], the existing techniques have been categorized in to five different types such as (a) Penalty methods, (b) Special representation and operator methods, (c) Repair methods, (d) Separation of objective and constraints methods and (e) Hybrid methods. However, in spite of many constraint-handling techniques exist in the literature; the penalty method is most commonly used by the researchers due to its efficient mechanism. This paper also uses the same penalty function method as follows. 1) The degree of constraint violation for an individual $x$ is evaluated by using the relation $G(x)=\frac{1}{m} \sum_{j=1}^{m} G_{j}(x)$ and

the degree of constrained violation is calculated by equation below

$$
G_{j}(x)=\left\{\begin{array}{l}
\max \left\{g_{j}(x), 0\right\} \quad 1 \leq j \leq q \\
\max \left\{\left|h_{j}(x)-\varepsilon\right|, 0\right\} \quad q+1 \leq j \leq m,
\end{array}\right.
$$

where $g_{i}(x)$ are the inequality constraints and $h_{j}(x)$ are the equality constraints present in the given optimization problem. $\varepsilon$ is a small positive tolerance value of equality constraints and is taken as $0.00001, q$ is the number of inequality constraints and $m-q$ is the equality constraints.

This is how the penalty method is being modified.

2) The fitness functions of each individual in the population then calculated by equation below.

$$
\begin{aligned}
& F(x)=f(x)+R\langle G(x)\rangle \\
& \text { where }\langle\alpha\rangle=\alpha, \text { if } \alpha \geq 0 \text { and otherwise zero. }
\end{aligned}
$$

$f$ and $R$ stands as the objective function and penalty parameter respectively. ' $R$ ' plays an important role to push the infeasible solutions towards feasible region. However, inappropriate value-assignment of $R$ may sometimes lead to produce absurd results. Hence the $R$ is fine tuned and a best suit value is proposed in Section V.

3) The tournament selection strategy is used to select the better fit individual between any two arbitrary individuals.

\section{DROSOPHILA FoOD-SEARCH CONSTRAINED OPTIMIZATION ALGORITHM}

Based on food-searching behavior of Drosophila
Melanogaster, a new algorithm is proposed in this section for finding global optimal solution of a non linear constrained optimization problem. It is named as Drosophila Food-Search Constrained Optimization Algorithm (DFCOA). Due to its highly sensitive sensory system including taste and olfaction, it is rich in the history of genetics. The olfactory receptors can sense all kinds of food sources whose scents blowing in the air and after getting the food sources, it can fly towards the foods and check the preference of the food sources by using gustatory receptors (GRs) present in the external sensilla of proboscis and then pass signal to the internal sensilla, whether it is allowed in digestive system or not. The internal sensilla of Drosophila comprises with GPCRs, a seven trans-membrane receptor that can sense molecules from outside the cell and activate the ligand binding [15]. The tendency of protein binding with the help of ligand depends on the concentration of ligand. If the ligand concentration is high, than GPCR will be activated and generate signal to the SOG region of the brain via nervous system and analysis took palace. If the input is more, then the SOG region automatically provides a positive signal to the proboscis to take food. Thus depending on the optimized food searching behavior of Drosophila the population based algorithm for solving of constrained problems is designed. The pseudo code of DFCOA algorithm is given below. begin

Gen $=1$

Generate initial population

Apply constrained Handling Technique

Evaluate fitness of each individual

While (termination criterion is not satisfied) do

Gen $=$ Gen +1

Apply Tournament selection (size 2)

Apply Redundant Search

Apply mQA

end do

end begin

\section{Computational Results}

The proposed algorithm is implemented in Dev $\mathrm{C}++$ on a Pentium Dual Core $2.00 \mathrm{GHz}$ machine with 1GB RAM under Windows7 platform. A total of 30 runs with population size 100 are conducted for each problem with maximum of 1 , 50,000 function evaluations as stopping criterion. To start a run, different seeds are used in order to generate the random numbers for the initial population. The proposed algorithm DFCOA is compared with a newly developed algorithm Hybrid Evolutionary Algorithm (HEA) [16], where the number of function evaluation used as 3,50,000 on 4 problems of g-Series and 6 problems of B-series as taken from[16] (given in Appendix- I \& II) .

After experimental results, the values of $\mathrm{R}$ for $\mathrm{g}$-Series and B-Series problems are fixed as 10000 . The results for 30 independent runs for $\mathrm{g}$-series problems are given in Table I and for B01-B06 are given in Table II with best, mean, worst and standard deviation.

From the results of Table I it is observed that g03, g05 and g11, DFCOA performs better than HEA, whereas for problem g13, the best achieved value by DFCOA is better 
than that of HEA. Mean, worse and standard deviation (SD) are not that good as HEA. However, from Table II, it is observed that for all test function from B01 to B06, DFCOA provides better function values than HEA. Also, DFCOA reaches the optimal value for all test functions with less SD.

TABLE I: THE COMPARATIVE RESUltS FOR PROBLEMS OF G-SERIES

\begin{tabular}{llllll}
\hline \hline Prob & Algo. & Best & Mean & Worst & Std Dev \\
\hline \multirow{2}{*}{ g03 } & HEA & $\mathbf{- 1 . 0 0 0}$ & $\mathbf{- 1 . 0 0 0}$ & $\mathbf{- 1 . 0 0 0}$ & $\mathbf{0 . 0 0 e + 0 0}$ \\
& DFCOA & $\mathbf{- 1 . 0 0 0}$ & $\mathbf{- 1 . 0 0 0}$ & $\mathbf{- 1 . 0 0 0}$ & $\mathbf{0 . 0 0 e + 0 0}$ \\
g05 & HEA & $\mathbf{5 1 2 6 . 4 9 9}$ & 5128.144 & 5137.793 & $5.21 \mathrm{e}+00$ \\
& DFCOA & $\mathbf{5 1 2 6 . 4 9 9}$ & $\mathbf{5 1 2 6 . 4 9 9}$ & $\mathbf{5 1 2 6 . 4 9 9}$ & $\mathbf{0 . 0 0 e + 0 0}$ \\
& HEA & $\mathbf{0 . 7 5}$ & $\mathbf{0 . 7 5}$ & $\mathbf{0 . 7 5}$ & $4.52 \mathrm{e}-09$ \\
& DFCOA & $\mathbf{0 . 7 5}$ & $\mathbf{0 . 7 5}$ & $\mathbf{0 . 7 5}$ & $\mathbf{0 . 0 0 e + 0 0}$ \\
& HEA & 0.053950 & $\mathbf{0 . 0 5 7 8 6 4}$ & $\mathbf{0 . 0 6 5 0 6 3}$ & $\mathbf{3 . 8 1 e - 0 3}$ \\
\hline \hline
\end{tabular}

TABLE II: THE COMPARATIVE RESULTS FOR PROBLEMS B01- B06

\begin{tabular}{cccccc}
\hline \hline Problems & Algorithm & Best & Mean & Worst & Std Dev \\
\hline \multirow{2}{*}{ B01 } & HEA & $\mathbf{- 4 7 . 6 2 7}$ & -46.988 & -46.497 & $3.50 \mathrm{e}-01$ \\
& DFCOA & $\mathbf{- 4 7 . 6 2 7}$ & $\mathbf{- 4 7 . 6 2 7}$ & $\mathbf{- 4 7 . 6 2 7}$ & $\mathbf{0 . 0 0 e + 0 0}$ \\
& & & & & \\
B02 & HEA & $\mathbf{9 6 1 . 7 1 5}$ & 961.717 & 961.721 & $1.71 \mathrm{e}-03$ \\
& & & & & \\
& DFCOA & $\mathbf{9 6 1 . 7 1 5}$ & $\mathbf{9 6 1 . 7 1 5}$ & $\mathbf{9 6 1 . 7 1 5}$ & $\mathbf{1 . 1 0 e - 0 3}$ \\
& & & & & \\
B03 & HEA & 194.113 & 205.921 & 288.427 & $7.87 \mathrm{e}+01$ \\
& & & & & \\
& DFCOA & $\mathbf{1 9 4 . 1 1 3}$ & $\mathbf{1 9 4 . 2 3 1}$ & $\mathbf{1 9 4 . 5 6 2}$ & $\mathbf{5 . 6 0 e - 0 1}$ \\
& & & & & \\
B04 & HEA & $\mathbf{0 . 0 0}$ & $\mathbf{0 . 0 0}$ & $\mathbf{0 . 0 0}$ & $\mathbf{0 . 0 0 e + 0 0}$ \\
& DFCOA & $\mathbf{0 . 0 0}$ & $\mathbf{0 . 0 0}$ & $\mathbf{0 . 0 0}$ & $\mathbf{0 . 0 0 e + 0 0}$ \\
& & & & & \\
B05 & HEA & 6.338 & 6.316 & 6.366 & $3.60 \mathrm{e}-02$ \\
& DFCOA & $\mathbf{6 . 3 3 8}$ & $\mathbf{6 . 3 4 0}$ & $\mathbf{6 . 3 6 2}$ & $\mathbf{2 . 1 6 e - 0 3}$ \\
& & & & & \\
& HEA & $\mathbf{- 1 . 0 0 0}$ & $\mathbf{- 1 . 0 0 0}$ & $\mathbf{- 1 . 0 0 0}$ & $1.78 \mathrm{e}-05$ \\
& DFCOA & $\mathbf{- 1 . 0 0 0}$ & $\mathbf{- 1 . 0 0 0}$ & $\mathbf{- 1 . 0 0 0}$ & $\mathbf{1 . 0 6 e - 0 9}$ \\
\hline \hline
\end{tabular}

Thus, from the comparative results it can be concluded that DFCOA performs overall better than HEA.

\section{REAL LIFE PROBLEMS}

In order to realize the efficiency of the DFCOA in solving real life problems, in this section, two real life problems namely Welded Beam Design-I, II and Pressure Vessel Design problem (marked as RP1-I, RP1-II and RP2, respectively) are considered. The results of DFCOA is compared with a newly developed algorithm called Hybrid Genetic Algorithm with Flexible Technique (GAFAT) [17], Differential Evolution Algorithm and Tissues P Systems (DEPTS) [18]. The population size for all the problems is fixed at 100 and average of best objective function value for all the 30 independent run with maximum function evaluation 6000 are reported in Table III.
From the results in Table III, it is concluded that DFCOA performs better than DEPTS and GAFAT in RP1-I and RP1-II both but for RP2, DEPTS performs better in comparison with DFCOA. However, it is here worth noting that the results reported in [17] and [18] are consisting with 20000 and 10000 function evaluations, whereas DFCOA uses only 6000 number of function evaluations. Usage of less number of function calls along with very small impact of SD confirms that DFCOA is a more stable algorithm and has faster rate of convergence.

TABLE III: THE COMPARATIVE RESULTS OF REAL LIFE PROBLEMS

\begin{tabular}{|c|c|c|c|c|c|}
\hline Probl. & Algo. & Best & Mean & Worst & Std Dev \\
\hline \multirow{3}{*}{ RP1-I } & GAFAT & 1.724852 & 1.724852 & 1.724852 & $5.80 \mathrm{e}-16$ \\
\hline & DEPTS & 1.724852 & 1.724852 & 1.724852 & $2.10 \mathrm{e}-07$ \\
\hline & DFCOA & 1.724852 & 1.724852 & 1.724852 & $4.59 \mathrm{e}-08$ \\
\hline \multirow[t]{2}{*}{ RP1-II } & GAFAT & $\underset{* * *}{2.380956}$ & $\underset{* * *}{2.380956}$ & $\underset{* * *}{2.380956}$ & 1.10e-15 \\
\hline & DFCOA & 2.380956 & 2.380956 & 2.380956 & $1.02 \mathrm{e}-07$ \\
\hline \multirow{3}{*}{ RP2 } & GAFAT & 6059.071 & 6059.0714 & 6059.071 & $2.80 \mathrm{e}+01$ \\
\hline & DEPTS & 5885.333 & $\mathbf{5 8 8 7 . 3 1 6}$ & 5942.323 & $1.0 \mathrm{e}+01$ \\
\hline & DFCOA & 6059.071 & 6059.0714 & 6059.071 & $0.00 \mathrm{e}+00$ \\
\hline
\end{tabular}

‘*’ denotes no results given.

\section{CONCLUSION}

This paper introduces a nature inspired algorithm called Drosophila Food-Search Constrained Optimization Algorithm (DFCOA). The proposed algorithm depends on the food-searching behavior of a fly Drosophila. To test the efficiency of DFCOA, a variety of 10 typical constrained optimization benchmark problems involving equality constrained have been solved. Further, two real life engineering problems have been solved by DFCOA. The statistical results and analysis concludes that the efficiency and efficacy of DFCOA is better than some state-of-the-art algorithms in solving not only to benchmark problems but also real life problems. DFCOA is robust and convergence faster with higher rate of stability. As a future scope of research, DFCOA may be applied to wide spectrum of engineering problems including robotics, electrical network, mechanical designs and manufacturing, etc. It can also be extended to solve multi-

\section{APPENDIX I}

$\mathrm{g}_{03}$

Minimize $f(x)=-(\sqrt{n})^{n} \prod_{i=1}^{n} x_{i}$

Subject to

$h(x)=\sum_{i=1}^{n} x_{i}-1=0$

where $n=10$ and $0 \leq x_{i} \leq 1(i=1, \ldots \ldots \ldots, n)$. 
$\mathrm{g}_{05}$

Minimize $f(x)=3 x_{1}+0.000001 x_{1}^{3}+2 x_{2}+(0.000002 / 3) x_{2}^{3}$

Subject to

$g_{1}(x)=-x_{4}+x_{3}-0.55 \leq 0$

$g_{2}(x)=-x_{3}+x_{4}-0.55 \leq 0$

$h_{3}(x)=1000 \sin \left(-x_{3}-0.25\right)+1000 \sin \left(-x_{4}-0.25\right)+894.8-x_{1}=0$

$h_{4}(x)=1000 \sin \left(x_{3}-0.25\right)+1000 \sin \left(x_{3}-x_{4}-0.25\right)+894.8-x_{2}=0$

$h_{5}(x)=1000 \sin \left(x_{4}-0.25\right)+1000 \sin \left(x_{4}-x_{3}-0.25\right)+1294.8=0$

where $0 \leq x_{1} \leq 1200,0 \leq x_{2} \leq 1200,-0.55 \leq x_{3} \leq 0.55$ and $-0.55 \leq x_{4} \leq 0.55$.

$\mathrm{g}_{11}$

Minimize $f(x)=-x_{1}^{2}+\left(x_{2}-1\right)^{2}$

Subject to

$h(x)=x_{2}-x_{1}^{2}=0$

where $-1 \leq x_{1} \leq 1,-1 \leq x_{2} \leq 1$.

$g_{13}$

Minimize $f(x)=e^{x_{1} x_{2} x_{3} x_{4} x_{5}}$

Subject to

$h_{1}(x)=x_{1}^{2}+x_{2}^{2}+x_{3}^{2}+x_{4}^{2}+x_{5}^{2}-10=0$

$h_{2}(x)=x_{2} x_{3}-5 x_{4} x_{5}=0$

$h_{3}(x)=x_{1}^{3}+x_{2}^{3}+1=0$

where $-2.3 \leq x_{i} \leq 2.3,(i=1,2)$ and $-3.2 \leq x_{i} \leq 3.2,(i=3,4,5)$.

\section{APPENDIX II}

$B 01$

Minimize $f(x)=\sum_{i=1}^{10} x_{i}\left(c_{i}+\ln \frac{x_{i}}{\sum_{j=1}^{10} x_{j}}\right)$

Subject to

$h_{1}(x)=x_{1}+2 x_{2}+2 x_{3}+x_{6}+x_{10}-2=0$

$h_{2}(x)=x_{4}+2 x_{5}+x_{6}+x_{7}-1=0$

$h_{3}(x)=x_{3}+x_{7}+x_{8}+2 x_{9}+x_{10}-1=0$

where $0 \leq x_{i} \leq 10,(i=1, \ldots \ldots \ldots, 10)$ and $c_{1}=-6.089, c_{2}=-17.164$,

$c_{3}=-34.054, c_{4}=-5.914 c_{5}=-24.721, c_{6}=-14.986$,

$c_{9}=-26.662, c_{10}=-22.179$.

$B 02$

Minimize $f(x)=1000-x_{1}^{2}-2 x_{2}^{2}-x_{3}^{2}-x_{1} x_{2}-x_{1} x_{3}$

Subject to

$h_{1}(x)=x_{1}^{2}+x_{2}^{2}+x_{3}^{2}-25=0$

$h_{2}(x)=8 x_{1}+14 x_{2}+7 x_{3}-56=0$

where $0 \leq x_{i} \leq 10$, $(i=1,2,3)$.
B03

Minimize $f(x)=x_{1}$

Subject to

$g_{1}(x)=-x_{1}+35 x_{2}^{0.6}+35 x_{3}^{0.6} \leq 0$

$h_{1}(x)=-300 x_{3}+7500 x_{5}-7500 x_{6}-25 x_{4} x_{5}+25 x_{4} x_{6}+x_{3} x_{4}=0$

$h_{2}(x)=100 x_{2}+155.365 x_{4}+2500 x_{7}-x_{2} x_{4}-24 x_{4} x_{7}-15536.5=0$

$h_{3}(x)=-x_{5}+\ln \left(-x_{4}+900\right)=0$

$h_{4}(x)=-x_{6}+\ln \left(x_{4}+300\right)=0$

$h_{5}(x)=-x_{7}+\ln \left(-2 x_{4}+700\right)=0$

where $0 \leq x_{1} \leq 1000,0 \leq x_{2}, x_{3} \leq 40,100 \leq x_{4} \leq 300,6.3 \leq x_{5} \leq 6.7$, $5.9 \leq x_{6} \leq 6.4$ and $4.5 \leq x_{7} \leq 6.25$.

B04

Minimize $f(x)=\left(x_{1}-1\right)^{2}+\left(x_{2}-x_{3}\right)^{2}+\left(x_{4}-x_{5}\right)^{2}$

Subject to

$h_{1}(x)=x_{1}+x_{2}+x_{3}+x_{4}+x_{5}-5=0$

$h_{2}(x)=x_{3}+2\left(x_{4}+x_{5}\right)+3=0$

where $0 \leq x_{i} \leq 2,(i=1,2, \ldots, 5)$.

B05

Minimize $f(x)=x_{1}+2 x_{2}+4 x_{5}+e_{1}^{x} x_{4}$

Subject to

$h_{1}(x)=x_{1}+2 x_{2}+5 x_{5}-6=0$

$h_{2}(x)=x_{1}+x_{2}+x_{3}-3=0$

$h_{3}(x)=x_{4}+x_{5}+x_{6}-2=0$

$h_{4}(x)=x_{1}+x_{4}-1=0$

$h_{5}(x)=x_{2}+x_{5}-2=0$

$h_{6}(x)=x_{3}+x_{6}-2=0$

where $0 \leq x_{i} \leq 2,(i=1,2, \ldots, 6)$.

B06

Minimize $f(x)=-x_{1}$

Subject to

$h_{1}(x)=x_{2}-x_{1}^{3}-x_{3}^{2}=0$

$h_{2}(x)=x_{1}^{2}-x_{2}-x_{4}^{2}=0$

where $0 \leq x_{i} \leq 2,(i=1, \ldots ., 4)$.

\section{WELDED BEAM DESIGN I}

Min

$C(h, l, t, b)=1.10471 h^{2} l+0.04811 t b(14.0+l)$

Subject to

$g_{1}(\tau)=13,600-\tau \geq 0, g_{2}(\sigma)=30,000-\sigma \geq 0$

$g_{3}(b, h)=b-h \geq 0, g_{4}\left(P_{c}\right)=P_{c}-6,000 \geq 0$

$g_{5}(\delta)=0.25-\delta \geq 0$

The expressions for $\tau, \sigma, \mathrm{P}_{\mathrm{C}}$ and $\delta$ are given by : 


$$
\begin{aligned}
& \tau=\sqrt{\left(\tau^{\prime}\right)^{2}+\left(\tau^{\prime \prime}\right)^{2}+\frac{l \tau \tau^{\prime}}{\alpha}}, \quad \alpha=\sqrt{0.25\left(l^{2}+(h+t)^{2}\right)}, \\
& \sigma=504000 /\left(t^{2} b\right), P_{c}=64746.022(1-0.0282346) t b^{3}, \\
& \delta=2.1952 /\left(t^{3} b\right), \tau^{\prime}=6000 /(\sqrt{2} h l)
\end{aligned}
$$

\section{WELDED BEAM DESIGN II}

$$
\operatorname{Min} f(x)=1.1047 x_{1}^{2} x_{2}+0.04811 x_{3} x_{4}\left(14+x_{2}\right)
$$

Subject to

$$
\begin{aligned}
& g_{1}(x)=\tau(x)-13600 \leq 0, g_{2}(x)=\rho(x)-30,000 \leq 0, g_{3}(x)=x_{1}-x_{4} \leq 0 \\
& g_{4}(x)=0.10471 x_{1}^{2}+0.04811 x_{3} x_{4}\left(14+x_{2}\right)-5.0 \leq 0 \\
& g_{5}(x)=0.125-x_{1} \leq 0, g_{6}(x)=\delta(x)-0.25 \leq 0 \\
& g_{7}(x)=6000-P_{c}(x) \leq 0, \text { where } \\
& \tau(x)=\sqrt{\left(\tau^{\prime}\right)^{2}+2 \tau^{\prime} \tau^{\prime \prime} \frac{x_{2}}{2 R}+\left(\tau^{\prime \prime}\right)^{2}}, \tau^{\prime}=\frac{6000}{\sqrt{2} x_{1} x_{2}}, \tau^{\prime \prime}=\frac{M R}{J} \\
& M=6000\left(14+\left(x_{2} / 2\right)\right), R=\sqrt{\frac{x_{2}^{2}}{4}+\left(\frac{x_{1}+x_{3}}{2}\right)^{2}} \\
& J=2\left(x_{1} x_{2} \sqrt{2}\left[\frac{x_{2}^{2}}{12}+\left(\frac{x_{1}+x_{3}}{2}\right)^{2}\right]\right) \\
& \sigma(x)=\frac{504000}{x_{4} x_{3}^{2}}, \delta(x)=\frac{65856000}{\left(30 \times 10^{6}\right) x_{4} x_{3}^{3}} \\
& P_{c}=\frac{4.013\left(30 \times 10^{6}\right) \sqrt{\frac{x_{3}^{2} x_{4}^{6}}{36}}}{196}\left(1-\frac{0.79056 x_{3}}{28}\right)
\end{aligned}
$$

$0.1 \leq x_{1}, x_{4} \leq 2$ and $0.1 \leq x_{2}, x_{3} \leq 10.0$

\section{PRESSURE VeSSEl Design}

$\operatorname{Min} W\left(T_{s}, T_{h}, R, L\right)=0.6224 T_{s} T_{h} R+1.7781 T_{h} R^{2}+3.1661 T_{s}^{2} L+19.84 T_{s}^{2} R$

Subject to

$$
\begin{aligned}
& g_{1}\left(T_{s}, R\right)=T_{s}-0.0193 R \geq 0, g_{2}\left(T_{h}, R\right)=T_{h}-0.00954 R \geq 0 \\
& g_{3}(R, L)=\pi R^{2} L+4 / 3 \pi R^{3}-1,296,000 \geq 0 \\
& g_{4}(L)=-L+240 \geq 0
\end{aligned}
$$

where, $0.0625 \leq T_{s}, T_{h} \leq 5, \quad 10 \leq R$, and $L \leq 200$

\section{REFERENCES}

[1] J. H. Hollad, Adaptive in Natural and Artificial Systems, The University of Michigan Press, Ann Arbor, MIT Press, 1975.
[2] J. H. Kennedy and R. C. Eberhart, "Particle swarm optimization," in Proc. International Conference on Neural Networks, 1995, pp. 1942-1948.

[3] L. A. Ingber, "Simulated annealing: practices versus theory," Journal of Mathematics and Computer Modeling, vol. 18, pp. 29-57, 1993.

[4] B. Franklin and M. Bergerman, "Cultural algorithms: concepts and experiments," in Proc. International Conference of IEEE Congress on Evolutionary Computation, 2000, pp. 1245-1251.

[5] A. W. Mohamed and H. Z. Sabry, "Constrained optimization based modified differential evolution algorithm," Information Sciences, vol. 194, pp. 171-208, July 2012.

[6] C. H. Lin, "A rough penalty genetic algorithm for constrained optimization," Information Sciences, vol. 241, pp. 119-137, August 2013.

[7] E. M. Montes and O. C. Dominguez, "Empirical analysis of a modified Artificial Bee Colony for constrained numerical optimization," Applied Mathematics and Computation, vol. 218, pp. 10943-10973, 2012.

[8] K. N. Das and T. K. Singh, "Drosophila food-search optimization algorithm," Applied Mathematics and Computation, vol. 231, pp. 566-580, 2014.

[9] V. G. Dethier, The Hungary Fly, Cambridge, MA: Harvard University Press.

[10] R. F. Stocker, "The organization of the chemosensory system in Drosophila melanogaster: a review," Cell Tissue Res, vol. 275, pp. 3-26, 1994.

[11] Q. Gaudry, K. I. Nagel, and R. I. Wilson, "Smelling on the fly: sensory cues and strategies for olfactory navigation in Drosophila," Current Opinion in Neurobiology, vol. 22, pp. 216-222, April 2012.

[12] Z. Michalewicz, "Evolutionary algorithm for constrained parameter optimization problem," Evolutionary Computation, vol. 4, no. 1, pp. $1-32,1996$.

[13] A. E. Eiben, "Evolutionary algorithms and constraint satisfaction: definitions, survey, methodology and research directions," in Theoretical Aspects of Evolutionary Computing, pp. 13-58, 2001.

[14] CAC Coello, "Theoretical and numerical constraint-handling techniques used with evolutionary algorithms: a survey of the state of the art computer methods," Applied Mechanics and Engineering, vol. 191, no. 11, pp. 1245-1287, 2002.

[15] H. Lodish, A. Berk, S. L. Zipursky, P. Matsudaira, D. Batlimore, and J. Darnell, Molecular Cell Biology: Cell Signalling, pp. 533-567, 2004.

[16] S. S. M. Abu, B. Ullah, R. Sarker, and C. Lokan, "Handling equality constraints in evolutionary optimization," European Journal of Operational Research, vol. 221, pp. 480-490, 2012.

[17] J. Zhao, L. Wang, P. Zeng, and W. Fan, "An efficient hybrid genetic algorithm with flexible allowance technique for constrained engineering design optimization," Expert Systems with Applications, vol. 39, pp. 6041-6051, April 2012

[18] G. Zhang, J. Cheng, M. Gheorghe, and Q. Meng, "A hybrid approach based on differential evolution and tissue membrane systems for solving constrained manufacturing parameter optimization problems," Applied Soft Computing, vol. 13, pp. 1528-1542, March 2013.

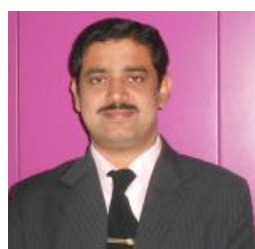

K. N. Das was born at Berhampur, Ganjam, Orissa, India. Dr. Das is currently an assistant professor in the Department of Mathematics at National Institute of Technology (NIT), Silchar, Assam (India). He achieved his Ph.D. degree from the Indian Institute of Technology, Roorkee, India, in 2008. During Ph.D., he has awarded MHRD scholarship from Govt. of India. He has around 30 publications. His current research interests is artificial intelligence and focuses on designing efficient hybrid algorithms to solve real life problems.

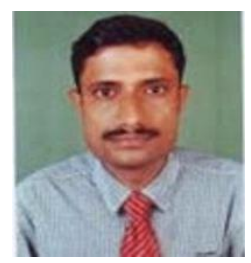

Tapan Kumar Singh was born in Silchar, India Mr. Singh has received his B.Sc degree (mathematics) from Cachar College, Silchar 1997 and completed M.Sc (mathematics) 1999 from Assam University, Silchar. He joined in National Institute of Technology, Silchar, India in 2010 as a research scholar. His research interests are soft computing and bio-inspired algorithms. 